\title{
A central performance drop with luminance stimuli requiring spatial integration
}

\author{
Cristina Meinecke \\ University of Erlangen-Nuremberg, Erlangen, Germany \\ AND \\ LOTHAR KEHRER \\ University of Bielefeld, Bielefeld, Germany
}

\begin{abstract}
This study reports four experiments that analyzed detection performance for luminance contrasts as a function of retinal eccentricity in order to find further support and explanations for the central performance drop (CPD) in the fovea. In the first experiment, 10 participants (16-37 years of age) had to detect a target patch in a stimulus consisting of bright and dark pixels. Luminance differences between target and context areas were achieved by placing a different number of bright (and dark) pixels in the target and the context area. Results showed a marked $\mathrm{CPD}$; that is, performance did not peak in the fovea but in the parafovea. A spatial integration hypothesis was proposed to explain this CPD. Alternative explanations were tested in three further experiments with a total of 28 participants from 19-46 years of age (using the decision criterion effect, the selective masking effect, and inhibition by high frequencies). The findings did not contradict the spatial-integration hypothesis.
\end{abstract}

When detection performance in early vision tasks is measured as a function of retinal eccentricity, it usually peaks in the fovea and declines with increasing eccentricity (see, e.g., Carrasco \& Yeshurun, 1998; Meinecke, 1989; Rovamo, Virsu, \& Näsänen, 1978). This effect is generally explained in terms of structural changes associated with increasing eccentricity within the visual system, such as decreasing density of receptors and/or increasing neural convergence and increasing receptive field size (for overviews, see Poirier \& Gurnsey, 2005; Strasburger, 2003).

However, there are cases in which a different pattern of results emerges, so that peak detection performance is no longer observed within the fovea, but within an area from $3^{\circ}-6^{\circ}$ of retinal eccentricity. In other words, performance first increases with increasing eccentricity and-after reaching a peak-declines with further eccentricity. This pattern of results is known as the central performance drop (CPD; Kehrer, 1987, 1989; see also, e.g., Gurnsey, Pearson, \& Day, 1996; Joffe \& Scialfa, 1995; Morikawa, 2000; Yeshurun \& Carrasco, 1998) and is mainly observed in texture segmentation tasks that use orientation differences between target and context elements.

One explanation for this CPD assumes that the visual system analyzes the stimulus with receptive fields or, more generally speaking, with spatial sampling units that differ in size depending on their retinal eccentricity: Within the foveal area, sampling units are small, but with increasing eccentricity they increase in size and, as a consequence, integrate information over a larger area (see, e.g., De Valois \& De Valois, 1988; Koenderink \& van Doorn, 1978). Detecting, for example, an orientation difference requires a spatial pooling of information. Thus, the detection of a target patch with elements that differ in their orientation from the context elements might benefit from the larger size of sampling units located in areas outside the fovea. We have termed this approach the spatialintegration hypothesis (see Meinecke \& Donk, 2002).

Furthermore, one must assume that in those tasks in which a CPD is observed, the visual system operates in a restricted mode and does not utilize larger sampling units located in the foveal region. This assumption is necessary because an inspection of the stimuli at longer exposure times reveals that we are not generally "blind" in the foveal region with regard to the detection of the target. This result coincides with the finding that larger receptive fields are also found in foveal regions (see, e.g., Rovamo et al., 1978). Hence, the CPD may be restricted to the specific experimental conditions in which it has been found (e.g., short presentation time, stimulus followed by a mask, suprathreshold target/context contrast).

The aim of the present study was to look for further evidence supporting the existence of the CPD and to explain its background. If the CPD can be traced back to structures of the visual system that are active during "early vision tasks," it should be possible to find a CPD for stimuli other than orientation contrast textures. We decided to construct stimuli meeting the following requirements: 
1. The stimuli or the task should be appropriate for early vision structures. Thus, our experimental task was to detect a luminance contrast (because we assume that detecting luminance contrast is a typical early vision task).

2 . The detection of the target should require some form of spatial integration. Thus, we constructed a stimulus consisting of bright and dark pixels (see Figure 1) so that the integrated luminance of the target patch was brighter than the integrated luminance of the context. This was achieved by placing a larger number of bright pixels in the target patch than in an equivalent context patch. Note that pixels could be either bright or dark, but that all bright pixels had the same bright luminance (and all dark pixels had the same dark luminance).

Our research question was now as follows: Because the perception of the "brighter" target required the integration of luminance information over a larger spatial area, would we find a CPD with these stimuli as well? We used the same presentation conditions that had revealed a CPD in earlier experiments: short presentation time, mask, and suprathreshold contrast between target and context.

\section{EXPERIMENT 1 Pixel Mask}

As described above, a stimulus was presented for a short duration to participants. The position of the target patch was varied on the horizontal meridian. The participants had to detect this target, which could appear on one-half of the trials. The stimuli were masked in order to ensure that performance was not at ceiling.

\section{Method}

Participants. Ten participants ( 4 male and 6 female) were paid to perform the experiment. They were 16-37 years old, and all had normal or fully corrected visual acuity. (Visual acuity was tested with a Rodenstock R22 Vision Tester using Test Stimuli No. 212; visual acuity was controlled in all experiments reported here.)

Apparatus. The experiment was run on a Power Macintosh G4 computer, with stimuli presented on an Iiyama HM 704 UTC screen at $85 \mathrm{~Hz}$. Stimulus presentation was controlled by a MATLAB program extended by the Psychophysics Toolbox (Brainard, 1997; Pelli,

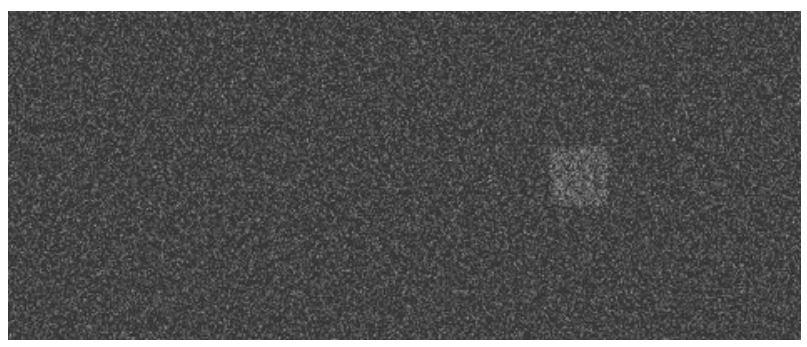

Figure 1.A sample stimulus for Experiment 1. The target patch varied its position along the horizontal meridian. Note that the luminance of the target patch has been increased slightly in this figure (in order to simulate the luminance conditions on the CRT display) by increasing the number of bright pixels in the target tiles to 150 (in the experiment, only 100 bright pixels were set in the target tiles).
1997). Luminance was measured with a Minolta luminance meter (Model LS-110).

Participants sat at a table with their heads in a head- and chinrest. The display monitor was positioned to give an observation distance of $45 \mathrm{~cm}$, with the direction of gaze inclined slightly downward. Participants responded to the stimuli by pressing one of two mouse keys with the index finger of either hand.

Stimuli. The stimulus consisted of (imaginary) tiles - 41 tiles in the horizontal direction and 17 in the vertical direction. Each tile was composed of $21 \times 21$ pixels (approximately $6.6 \times 6.6 \mathrm{~mm}$, or $0.81^{\circ} \times 0.81^{\circ}$ of visual angle). Thus, the stimulus field subtended $33.2^{\circ}(270 \mathrm{~mm})$ in the horizontal and $13.7^{\circ}(112 \mathrm{~mm})$ in the vertical direction. The target patch consisted of $3 \times 3$ tiles and could appear on one of the central 25 positions on the horizontal meridian of the stimulus field. The maximum eccentricity of the target (in terms of the center of the target patch) was $9.71^{\circ}$ to the left or right of the center of the stimulus field.

The context tiles consisted of 60 bright $\left(27 \mathrm{~cd} / \mathrm{m}^{2}\right)$ and 381 dark $\left(2 \mathrm{~cd} / \mathrm{m}^{2}\right)$ pixels, distributed randomly over the tile area. This resulted in an integrated luminance of approximately $5.0 \mathrm{~cd} / \mathrm{m}^{2}$. The target tiles consisted of 100 bright and 341 dark pixels (same luminances as the context pixels). This resulted in an integrated luminance of approximately $7.0 \mathrm{~cd} / \mathrm{m}^{2}$. The mask, which appeared after stimulus presentation, was the same size as the stimulus. Each tile of the mask consisted of 80 bright pixels and 361 dark pixels, distributed randomly across the tile area. This resulted in an integrated luminance of $6.0 \mathrm{~cd} / \mathrm{m}^{2}$. The background of the screen had a luminance of $13 \mathrm{~cd} / \mathrm{m}^{2}$.

Procedure. A block consisted of $50(2 \times 25)$ positive trials, in which a target was presented twice at each of the 25 possible positions, and 50 negative trials, in which the stimulus field contained no target. The sequence of positive and negative trials and the target position in each positive trial were randomized.

Each stimulus presentation was preceded by a small circle displayed at screen center informing the participant that the computer was ready and that he or she could summon the stimulus display by simultaneously pressing both mouse keys. The computer then replaced the circle by a fixation point $(2 \times 2$ pixels $)$, which was replaced automatically by the stimulus after $800 \mathrm{msec}$. Stimuli were displayed for $23.5 \mathrm{msec}$ and then replaced by the mask. The mask remained on the screen until the participant responded by pressing either the left (no target present) or the right key (target present). A short, single acoustic click informed the participant that a response was correct; a short double click, that he or she had made an error. Then the circle was displayed on the screen again, indicating that a new trial could be initiated.

Participants were instructed to focus on the fixation point as closely as possible, to respond as quickly as possible, and to make as few false alarms as possible. The last point was introduced to keep false alarms at a minimum, so that a participant would only give a "yes" response if he or she was relatively certain that the stimulus contained a target. The reason for this was to keep individual differences in criterion as low as possible. Because the hit rate varied as a function of the retinal eccentricity of the target and because the position of the target varied randomly within a block, participants could define their criterion only in relation to the negative trials (those without a target). As Treisman and Watts (1966; see also Neyman $\&$ Pearson, 1933) proposed, it makes sense to use the false alarm rate in the instructions to influence participants to set their criterion for such an experimental situation, in which signal strength varies within the experimental condition.

Two sessions were administered, each lasting about $40 \mathrm{~min}$. At the beginning of Session 1, two practice blocks with only one target per position were presented. In the first block, the stimuli were presented for $47 \mathrm{msec}$, and in the second, they were presented for $23.5 \mathrm{msec}$. Before Session 2, one practice block was presented, with a presentation time of $23.5 \mathrm{msec}$. Data from the practice blocks were not analyzed further. After the practice blocks, two blocks were presented in Session 1 and five blocks in Session 2. 
Experiment 1

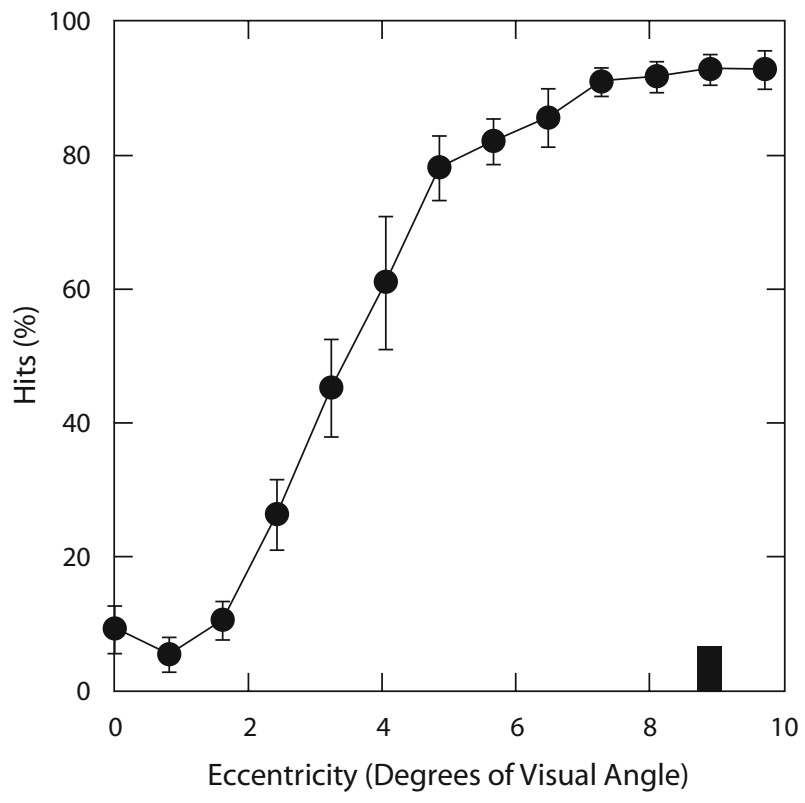

Figure 2. Experiment 1 (pixel mask): Hits as a function of the retinal eccentricity of the target. The bar at lower right shows the percentage of false alarms.

\section{Results and Discussion}

For each participant, all trials in which the reaction time exceeded the mean for that block by three standard deviations were dropped from further analyses. We applied this procedure in all experiments.

Figure 2 presents the percentage of hits as a function of the eccentricity of the target (as well as the percentage of false alarms, 6.53\%). An ANOVA for repeated measures was calculated with the variable position (1-13; we averaged results for the corresponding left and right positions). There was a significant effect of position $[F(12,108)=$ $89.05, p<.001]$. An alpha level of .05 was used for all statistical tests.

The results showed a marked CPD: Targets were detected with a high probability at the most eccentric positions, but detection performance declined rapidly when approaching the fovea. Thus, the results seem to fit into the context of the spatial-integration hypothesis. However, we first have to exclude several alternative explanations for this pattern of results:

1. Response criterion shift. The performance drop in the foveal area may have been due to a shift in response criterion by the participants (in the direction of more conservative response behavior), and not to a shift in sensitivity. Although Gurnsey et al. (1996), for example, showed that this was not the case in their conditions, it is still necessary to ensure that this also holds for luminance stimuli. Therefore, in Experiment 2 we collected data on the false alarm rate per target position in order to be able to calculate $d^{\prime}$.

2. Selective foveal masking by pixel structure. The mask used in Experiment 1 consisted of bright and dark pixels. One could argue that this mask contains high frequen- cies, and that these may have failed to mask the stimulus uniformly. Because the fovea is more sensitive to high frequencies than the periphery, the mask may have had a stronger impact in foveal than in peripheral areas. Experiment 3 tested this by using a homogeneous mask.

3. Cross-frequency inhibition. The pixel structure used in our stimuli contained high frequencies, but such high frequencies are not informative for the detection of the luminance contrast. Now, as Gurnsey et al. (1996) commented already, it may be that when a stimulus contains high frequencies, these are processed preferentially. Because foveal regions are particularly sensitive to high frequencies, which our stimuli contained, we cannot rule out the possibility that the CPD was due to a so-called cross-frequency inhibition, in which high-frequency processing in the fovea "inhibits" the processing of the lower frequencies that presumably form the basis for detecting the luminance target. Experiment 4 tested this assumption by applying - in accordance with Gurnsey et al. (1996) low-pass filtering as a manipulation.

\section{EXPERIMENT 2 Calculation of $d^{\prime}$}

In this experiment, we wanted to obtain information on the hit and false alarm rates for specific target positions. The aim was to calculate $d^{\prime}$ in order to ensure that the CPD is based on differences in sensitivity and not in response criterion behavior. We chose five different target eccentricities that were presented as different conditions.

\section{Method}

Participants. Ten participants ( 9 female and 1 male) were paid to participate in the experiment. They were 19-26 years old.

Apparatus. This was identical to that in Experiment 1.

Stimuli. Both the stimuli and the mask were identical to those in Experiment 1, with one exception: We used only stimuli with targets at five different eccentricities. These eccentricities were Position 1 $\left(0^{\circ}\right)$, Position $2\left(1.62^{\circ}\right)$, Position $3\left(3.24^{\circ}\right)$, Position $4\left(5.67^{\circ}\right)$, and Position $5\left(8.10^{\circ}\right)$. In Positions 2-5, targets were presented both to the left and to the right of the center of the stimulus.

Procedure. The five eccentricity conditions (Positions 1-5) were blocked. A "position" block consisted of 50 positive trails (25 lefthand and 25 right-hand targets, or 50 central targets in Position 1) and 50 negative trials (in which the stimulus field contained no target). The sequence of positive and negative trials, as well as the target position in the positive trials (left vs. right), was randomized.

Two sessions were administered, each lasting about $40 \mathrm{~min}$. At the beginning of Session 1, two practice blocks were presented (stimulus presentation time: $23.5 \mathrm{msec}$ ). These practice blocks contained 10 positive and 10 negative trials, with two eccentricities chosen (Positions 2 and 4). In Session 2, one practice block was presented (Position 3).

After the practice blocks, the five position conditions were presented successively in both sessions (stimulus presentation time: $23.5 \mathrm{msec}$ ). Participants were instructed to miss as few targets as possible and to produce as few false alarms as possible. All other details were identical to those of Experiment 1.

\section{Results and Discussion}

Hit rates were averaged for left- and right-hand target positions in each position condition (2-5). The $d^{\prime}$ values in Figure 3 show that the variation of eccentricity im- 


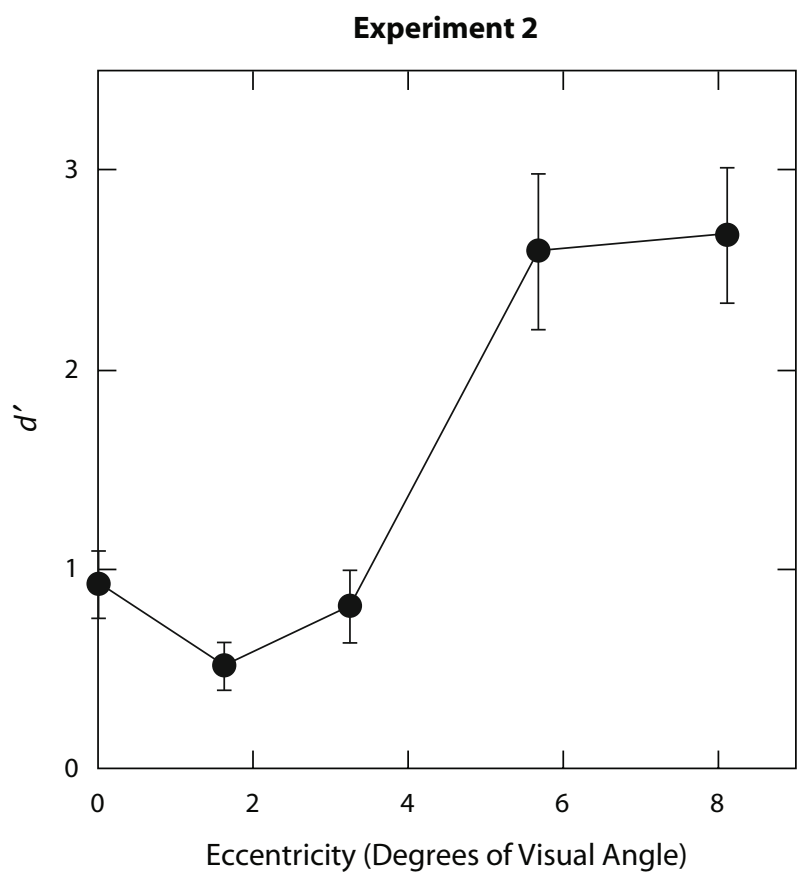

Figure 3. Experiment 2: $d^{\prime}$ values as a function of the retinal eccentricity of the target.

pacted on sensitivity. An ANOVA for repeated measures was calculated with position (1-5) as the variable, revealing a significant effect of position $[F(4,36)=16.50, p<$ $.001]$. Thus, the CPD could be replicated, indicating that the CPD is not attributable to differences in response criterion alone, but to differences in sensitivity.

There seemed to be a slight performance increase when the target was displayed at the central position. One could think of an effect of position certainty or uncertainty: Unlike in Experiment 1, in which all positions of the target were presented in a random order, in the $0^{\circ}$ condition of Experiment 2 there was no position uncertainty; the target always appeared at the center. This was not the case in the other four conditions, in which the target could appear to the left or to the right of the fixation point. This perfect predictability of the target position may have facilitated its detection. The central condition here was similar to a situation in which performance is improved by a valid cue (see Posner, 1980). A statistical analysis revealed no significant performance increase, however, in the central position relative to the adjacent position (Wilcoxon test). Therefore, we will not speculate further on this pattern of results.

\section{EXPERIMENT 3 Homogeneous Mask}

Experiment 3 replicated Experiment 1, with one exception: We used a homogeneous mask (instead of a pixel mask). If the high frequencies contained in the pixel mask impaired detection performance in the foveal region selectively, then the CPD should disappear if a homogeneous mask was used. ${ }^{1}$ The first evidence that a noise (or pixel) mask per se does not produce a CPD was reported by Gurnsey, Di Lenardo, and Potechin (2004), who analyzed texture segmentation performance with line elements that differed in orientation. When the researchers used a mask with X-like elements, they observed a pronounced CPD. When they used a noise mask, foveal performance actually improved (and peripheral performance deteriorated). In that condition, the CPD disappeared almost completely. On the other hand, Potechin and Gurnsey (2003) already showed that a CPD - found with oriented texture elements-remained observable even when no mask followed the stimulus.

\section{Method}

Participants. Ten participants (all female) were paid to participate in the experiment. They were 19-31 years old.

Apparatus, Stimuli, and Procedure. These were identical to their counterparts in Experiment 1, with the following exception: The mask was a homogeneous stimulus with a luminance of $6.0 \mathrm{~cd} / \mathrm{m}^{2}$.

\section{Results and Discussion}

Figure 4 presents the percentage of hits as a function of target eccentricity (as well as the percentage of false alarms, $1.37 \%$ ). An ANOVA for repeated measures calculated with the variable position (1-13) revealed a significant effect $[F(12,108)=22.91, p<.001]$.

Again, the slight increase in performance at the central position was not significant in comparison with the adjacent position (Wilcoxon test).

A homogeneous mask (rather than a pixel mask) made detection performance less difficult. However, once again,

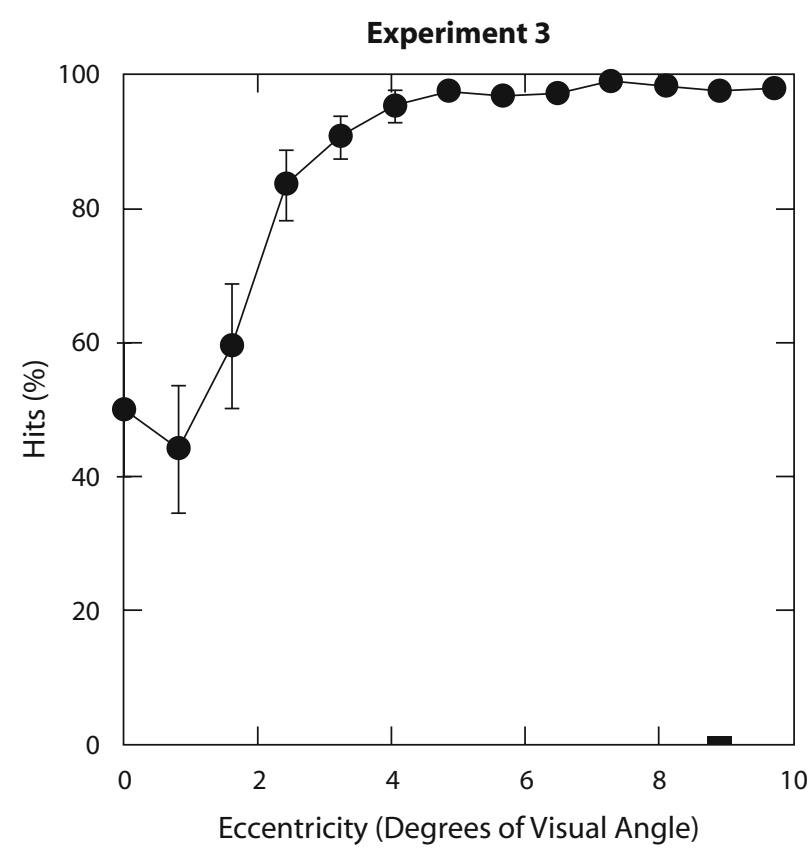

Figure 4. Experiment 3 (homogeneous mask): Hits as a function of the retinal eccentricity of the target. The bar shows the percentage of false alarms. 


\section{Experiment 4}

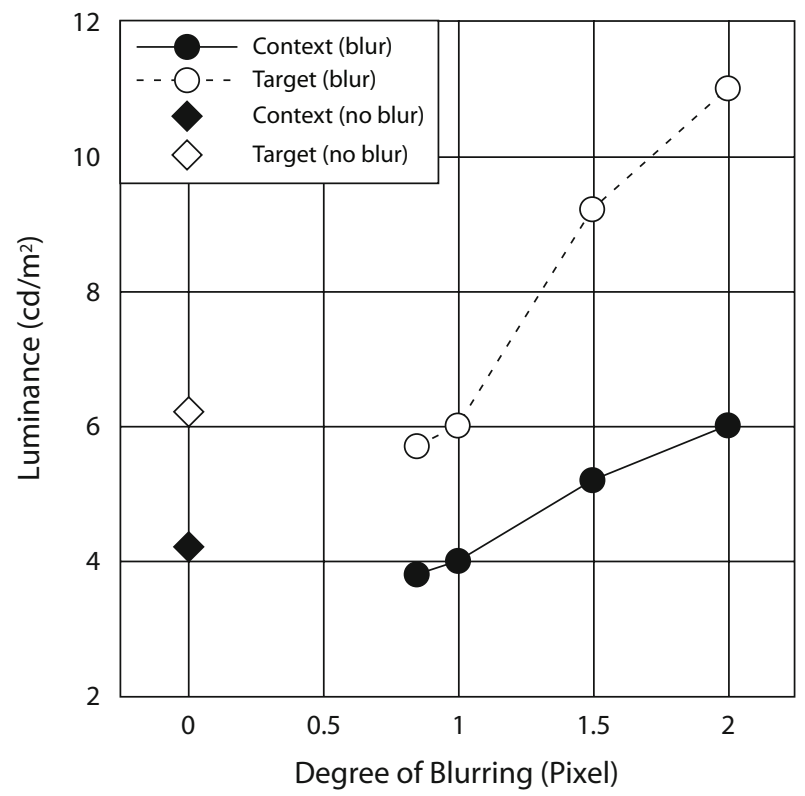

Figure 5. Experiment 4: Luminance of the target and context tiles in the no-blur condition and with four different degrees of blurring (low-pass filtered with a Gaussian kernel, sigma $=x$ pixels). A blurring with sigma $=1$ pixel leaves luminances nearly unchanged in comparison with the no-blur condition.

performance dropped when the target was displayed in the central positions, replicating the CPD from Experiment 1. Thus, the CPD found in Experiment 1 cannot be attributed just to the pixel mask used in that experiment.

\section{EXPERIMENT 4 \\ Cross-Frequency Inhibition: Low-Pass Filtering of Stimuli}

Experiment 4 tested whether the CPD is a consequence of a cross-frequency inhibition in the foveal region (see Gurnsey et al., 1996). Low-frequency stimulus information, which presumably is informative and necessary to detect the target, is inhibited by the presence of highfrequency information (the pixels in the stimulus); with increasing eccentricity, the dominance or existence of high-frequency processors diminishes, and thus conflicting information is reduced. Note that this time we focused on stimulus properties, whereas in Experiment 3 we focused on the mask.

In analogy to a manipulation performed by Gurnsey et al. (1996), we blurred the stimulus. If the CPD is a consequence of an interfering effect of the stimulus high frequencies within the foveal area, a blurring or low-pass filtering of the stimulus should have a differential effect on detection performance, in which performance in the foveal area should profit more from blurring than should performance in peripheral areas.

Two conditions were realized in this experiment. In the pixel condition, stimuli were identical to those in Experi- ment 1; in the blur condition, the stimuli in the pixel condition were low-pass filtered.

\section{Method}

Participants. Eight participants (all female) were paid to participate in the experiment. They were 19-46 years old (mean, 29.13 years).

Stimuli. The stimuli in the pixel condition were identical to the stimuli in Experiment 1. The stimuli in the blur condition were identical to those in the pixel condition, with one exception: They were moderately low-pass filtered with a Gaussian kernel. To be sure that the blurring procedure did not alter the luminances of the stimuli, in a first step we systematically varied the degree of blurring. Figure 5 shows the results: With increasing blurring (sigma of the Gaussian kernel, in pixels), the luminance of the stimulus increased, and the difference between target and context luminance increased as well. With a sigma of 1 pixel, the luminances of the blurred and unblurred stimuli were very similar. Therefore, the stimuli used in the blur condition were low-pass filtered with a sigma of 1 pixel (Figure 6).

The background of the display and the homogeneous mask had a luminance of $12 \mathrm{~cd} / \mathrm{m}^{2}$. This was slightly higher than in Experiment 3 , because performance in a pilot experiment had been close to ceiling. This increase in mask luminance made the task more difficult.

Apparatus and Procedure. These were identical to those in Experiment 1, with the following exception: Whereas the presentation time of the stimuli was once again $23.5 \mathrm{msec}$ for 2 of the participants, it was reduced to $11.7 \mathrm{msec}$ for the other 6 , in order to prevent their performance from being at ceiling.

\section{Results and Discussion}

Figure 7 presents the percentage of hits as a function of target eccentricity. An ANOVA for repeated measures was calculated with the variables condition (pixel vs. blur) and position (1-13). In the blurred condition, targets were detected significantly more frequently than in the pixel condition $[F(1,7)=82.60, p<.001]$. There was a significant effect of position $[F(12,84)=19.31, p<.001]$, and also a significant interaction between condition and position $[F(12,84)=2.06, p<.05]$.

In the pixel condition, more false alarms were made than in the blurred condition $(6.00 \%$ vs. $3.32 \%)$. This difference allows us to interpret the difference in the hit rates between the two conditions, because a higher hit rate is coupled with a lower false alarm rate (and vice versa).

To test whether the significant interaction between condition and position was caused by a selective influ-

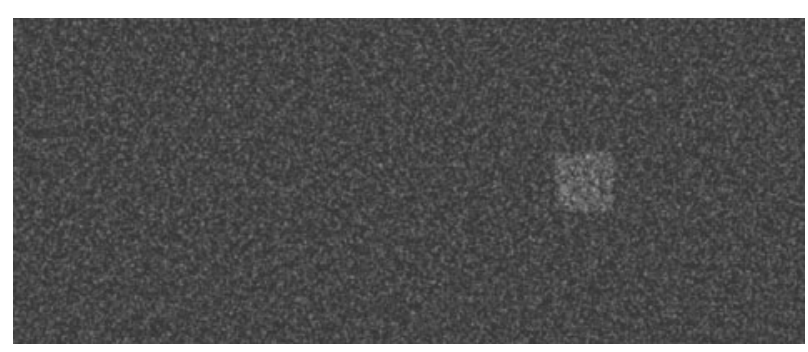

Figure 6. A sample stimulus for the blur condition in Experiment 4. Note that the luminance of the target patch has been increased slightly in this figure (in order to simulate the luminance conditions on the CRT display) by increasing the number of bright pixels in the target tiles from 100 to 150 . 


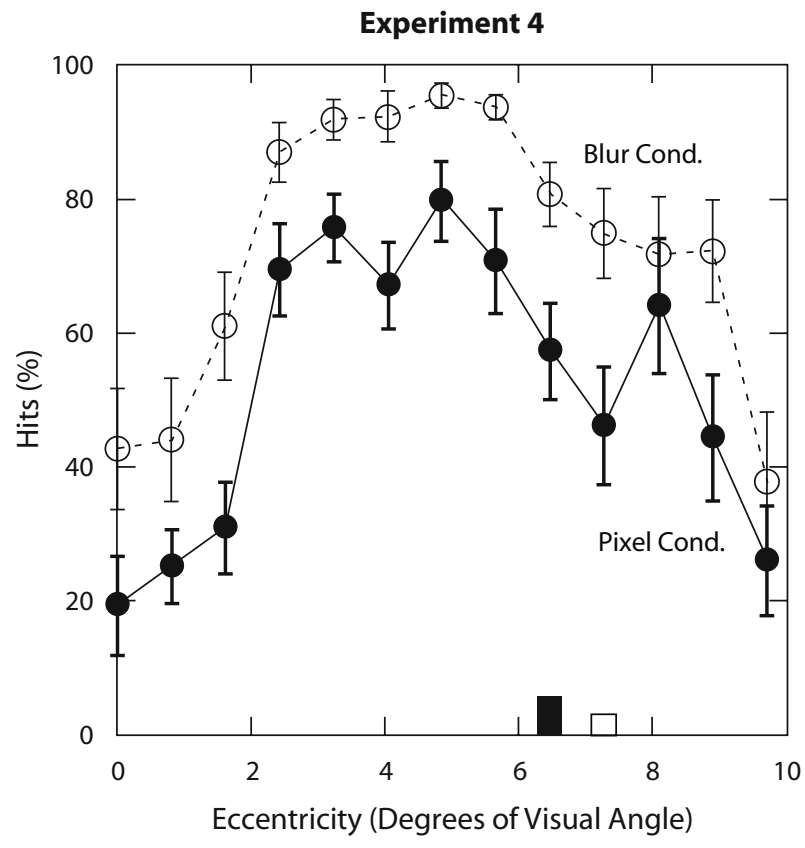

Figure 7. Experiment 4 (low-pass filtering): Hits as a function of the retinal eccentricity of the target for the pixel condition (filled circles) and the blur condition (open circles). The bars show percentages of false alarms for the two conditions.

ence of the blurring manipulation on the foveal region, we computed the difference between the two conditions and submitted these difference data to a trend analysis. Neither the linear nor the quadratic trend showed a significant result [linear, $F(1,7)=0.792$, n.s.; quadratic, $F(1,7)=$ 0.731 , n.s.]. Thus, in the low-pass-filtered condition, targets could generally be detected better than in the pixel condition, but without a specific eccentricity trend. This means that the CPD does not seem to be the result of a cross-frequency inhibition in the foveal region.

Note that, contrary to Gurnsey et al. (1996), the blurring of the stimulus led to a general increase in performance, not to the decrease they demonstrated with texture stimuli. Their orientation discrimination task needed some amount of high-frequency information, whereas our luminance contrast detection task profited from the blurring manipulation. This performance increase through blurring can be seen as further evidence that detecting the target did not require high frequencies, and presumably was based on lower frequencies; when high frequencies were reduced, the overall visibility of our target patch increased. High frequencies impair detection performance in this task independently of eccentricity. In the General Discussion, we speculate about why blurring improves detection performance in this task.

Unlike in Experiments 1, 2, and 3, detection performance in Experiment 4 dropped in the periphery. We assume that this goes back to the fact that the task was more difficult in the present experiment than in the previous experiments. In Experiment 4, our aim was to avoid any form of ceiling effect that might have hidden interaction effects with eccentricity. As the data of Experiments 1-3 show, peripheral performance was nearly at ceiling in those experiments. To increase the difficulty of the task, we increased the luminance of the mask (in Experiments $1-3,6 \mathrm{~cd} / \mathrm{m}^{2}$; in Experiment 4, $12 \mathrm{~cd} / \mathrm{m}^{2}$ ). Moreover, for 6 of the 8 participants we reduced the presentation time from $23.5 \mathrm{msec}$ (as in Experiments 1-3) to $11.7 \mathrm{msec}$. These manipulations led to a performance decrement. Moreover, we know that there is a marked variation between participants in the size of their useful field of view in this sort of task. Therefore, we cannot exclude that the peripheral drop in this experiment relates back to individual differences.

\section{GENERAL DISCUSSION}

The aims of the present study were to look for further evidence for the existence of the CPD and to explain its background. We analyzed luminance contrast detection performance as a function of retinal eccentricity and used stimuli that required a spatial integration of luminance information in order to achieve target detection. Our first experiment demonstrates a clear CPD, thus delivering some first evidence favoring the spatial-integration hypothesis.

The following experiments tested alternative explanations for the CPD. Experiment 2 showed that the CPD is not attributable to a response criterion shift, but that sensitivity in the foveal region is actually reduced relative to that in peripheral positions. Experiment 3 showed that the CPD cannot be attributed solely to a selective foveal masking effect from the high-frequency content of the pixel mask: A homogeneous mask facilitated the task, but did not eliminate the CPD. Finally, Experiment 4 showed that the CPD is not a result of a cross-frequency inhibition in the foveal region by the high-frequency content of the stimulus itself, since a low-pass-filtered version of the stimulus had no selective effect on the CPD.

In sum, the experiments show that a CPD can be observed not only with textures with an orientation difference between the target and context elements, but also in a luminance contrast detection task. Our luminance task required the spatial integration of luminance information; an analysis of the luminance of single pixels would not help to detect the target. Therefore, the data support the spatial-integration hypothesis as an explanatory background for the CPD. The data suggest that under the presentation conditions used here, the visual system operates in a restricted mode: A "switch" to larger spatial-sampling units in the foveal region does not take place, even though this would facilitate task performance. A look at the stimulus (see Figure 1) shows that the fovea is not incapable of seeing the target patch.

Nonetheless, some questions remain open:

1. The assumption of a restricted operation mode is necessary, because Rovamo et al. (1978) showed that the detection of luminance contrast is always better in the fovea than in the periphery. In contrast to our study, Rovamo et al. displayed their stimuli for $0.5 \mathrm{sec}$ and measured contrast sensitivity at threshold. One or both of these factors in their design may be responsible for the different results 
between their study and ours. A systematic variation of these factors (presentation time and amount of luminance contrast) could be informative in bridging the gap between these diverging patterns of results.

2. The detrimental influence of high frequencies irrespective of eccentricity (see Experiment 4 ) requires further analysis. It looks as if the detection mechanism works more effectively in the blurred condition. Blurring might not so much influence the encoding of the stimulus (which should have an eccentricity-specific effect) as influence the detection or decision mechanism, in that a reduction of the noise signal helps participants to distinguish the relevant signal. Such an effect of noise (or of its reduction or elimination) has been shown in continuous visual search experiments, in which search times and false alarm rates are reduced significantly in a no-noise condition relative to a noise condition (Prinz, Meinecke, $\&$ Hielscher, 1987). This decision mechanism explanation (in contrast to an encoding mechanism explanation) is speculative at this time, but it offers a promising direction for future research.

The focus of this study has been an analysis of the CPD. We have tried to rule out the possibility that the observed CPD is an artifact of response criterion, masking, or crossfrequency inhibition. Indeed, the CPD resists all manipulations, thus suggesting that the explanatory background of the spatial-integration hypothesis may be both useful and appropriate.

\section{AUTHOR NOTE}

This research was supported by Grants ME 1656/2-1 and KE 388/4-1 from the Deutsche Forschungsgemeinschaft. We thank Stefanie Harbich and Julia Tasche for their help in running the experiments, and Jonathan Harrow for native-speaker advice. Correspondence relating to this article may be sent to C. Meinecke, Institute of Psychology, University of Erlangen-Nürnberg, Kochstr. 4, 91054 Erlangen, Germany (e-mail: meinecke@rzmail.uni-erlangen.de) or to L. Kehrer, Department of Psychology, University of Bielefeld, Universitätsstraße 25, 33501 Bielefeld, Germany (e-mail: lothar.kehrer@uni-bielefeld.de).

\section{REFERENCES}

Brainard, D. H. (1997). The Psychophysics Toolbox. Spatial Vision, 10, $433-436$.

Carrasco, M., \& Yeshurun, Y. (1998). The contribution of covert attention to the set-size and eccentricity effects in visual search. Journal of Experimental Psychology: Human Perception \& Performance, 24, 673-692.

De Valois, R. L., \& De Valois, K. K. (1988). Spatial vision. New York: Oxford University Press.

Gurnsey, R., Di Lenardo, D., \& Potechin, C. (2004). Backward masking and the central performance drop. Vision Research, 44, 2587-2596.

Gurnsey, R., Pearson, P., \& Day, D. (1996). Texture segmentation along the horizontal meridian: Nonmonotonic changes in perfor- mance with eccentricity. Journal of Experimental Psychology: Human Perception \& Performance, 22, 738-757.

Joffe, K. M., \& Scialfa, C. T. (1995). Texture segmentation as a function of eccentricity, spatial frequency and target size. Spatial Vision, 9, 325-342.

KeHRER, L. (1987). Perceptual segregation and retinal position. Spatial Vision, 2, 247-261.

KeHRER, L. (1989). Central performance drop on perceptual segregation tasks. Spatial Vision, 4, 45-62.

KoenderinK, J. J., \& VAn Doorn, A. J. (1978). Visual detection of spatial contrast: Influence of location in the visual field, target extent and illuminance level. Biological Cybernetics, 30, 157-167.

MeINECKE, C. (1989). Retinal eccentricity and the detection of targets. Psychological Research, 51, 107-116.

Meinecke, C., \& DonK, M. (2002). Detection performance in pop-out tasks: Nonmonotonic changes with display size and eccentricity. Perception, 31, 591-602.

Morikawa, K. (2000). Central performance drop in texture segmentation: The role of spatial and temporal factors. Vision Research, 40, 3517-3526.

Neyman, J., \& Pearson, E. S. (1933). On the problems of the most efficient tests of statistical hypotheses. Philosophical Transactions of the Royal Society of London: Series A, 231, 289-338.

PelLi, D. G. (1997). The VideoToolbox software for visual psychophysics: Transforming numbers into movies. Spatial Vision, 10, 437-442.

Poirier, F. J. A. M., \& Gurnsey, R. (2005). Non-monotonic changes in performance with eccentricity modeled by multiple eccentricitydependent limitations. Vision Research, 45, 2436-2448.

PosNer, M. I. (1980). Orienting of attention. Quarterly Journal of Experimental Psychology, 32, 3-25.

PoteChin, C., \& GuRnSEY, R. (2003). Backward masking is not required to elicit the central performance drop. Spatial Vision, 16, 393-406.

Prinz, W., Meinecke, C., \& Hielscher, M. (1987). Effect of stimulus degradation on category search. Acta Psychologica, 64, 187-206.

Rovamo, J., VIRSU, V., \& NÄSÄNEN, R. (1978). Cortical magnification factor predicts the photopic contrast sensitivity of peripheral vision. Nature, 271, 54-56.

SPERLING, G. (1960). The information available in brief visual presentation. Psychological Monographs: General \& Applied, 74(Whole No. 498), 1-29.

Strasburger, H. (2003). Indirektes Sehen: Formerkennung im zentralen und peripheren Gesichtsfeld [Indirect vision: Shape recognition in the central and peripheral visual field]. Göttingen: Hogrefe.

Treisman, M., \& WatTs, T. R. (1966). Relation between signal detectability theory and the traditional procedures for measuring sensory thresholds: Estimating $d^{\prime}$ from results given by the method of constant stimuli. Psychological Bulletin, 66, 438-454.

Yeshurun, Y., \& CARrasco, M. (1998). Attention improves or impairs visual performance by enhancing spatial resolution. Nature, 396, $72-75$.

\section{NOTE}

1. Note that a homogeneous mask could also be described as "no mask" and may decrease the overall difficulty of the task. But as Sperling (1960) already noted, even a blank screen can act as a mask.

(Manuscript received March 18, 2004; revision accepted for publication January 8, 2007.) 